\title{
Editorial
}

\section{Uncertain Dynamical Systems: Analysis and Applications}

\author{
Gani Stamov, ${ }^{1}$ Haydar Akca, ${ }^{2}$ and Ivanka Stamova ${ }^{3}$ \\ ${ }^{1}$ Department of Mathematics, Technical University of Sliven, 8800 Sliven, Bulgaria \\ ${ }^{2}$ Department of Applied Sciences and Mathematics, Abu Dhabi University, College of Arts and Sciences, P.O. Box 59911, \\ Abu Dhabi, UAE \\ ${ }^{3}$ Department of Mathematics, University of Texas at San Antonio, San Antonio, TX 78249, USA
}

Correspondence should be addressed to Gani Stamov; gstamov@abv.bg

Received 22 April 2013; Accepted 22 April 2013

Copyright ( $\odot 2013$ Gani Stamov et al. This is an open access article distributed under the Creative Commons Attribution License, which permits unrestricted use, distribution, and reproduction in any medium, provided the original work is properly cited.

Many real word phenomena exist under the conditions of structural uncertainty. Also, in many applications of dynamical systems the uncertainties happen frequently due to modeling errors, measurement inaccuracy, mutations in the evolutionary processes, and so on.

The dynamic of uncertain systems has long been and will continue to be one of the dominant themes in mathematics and mathematics applications due to its theoretical and practical significance. During the last couple of decades, analysis of uncertain dynamical systems and related models has attracted the attention of a wide audience of professionals such as mathematicians, applied researches, and practitioners. In spite of the amount of published results recently focused on such systems, there remain many challenging open questions. The theory and applications of these systems are still very active areas of research.

In this special issue, we have invited a few papers that address such issues.

Ten papers deal with some theoretical aspects of dynamical systems under uncertainties. The stabilization of dynamic switched control systems is explored by M. De la Sen using an operator-based formulation. H. Akhadkulov and M. S. Md Noorani, focusing on a consideration of theory of marginal, provide one sufficient and one necessary condition for the absolute continuity of conjugation map between circle maps with break points. The paper by T. Allahviranloo et al. describes a new approach for determining the general solutions of a fully fuzzy linear system. Examples are discussed to illustrate the main results. S. He studies a filtering problem for a class of uncertain Markovian jumping systems within the finite-time interval. A sufficient condition is derived such that the filtering dynamic error from the unknown input is finite time bounded and satisfies a prescribed level of disturbance attenuation in a finite time interval. The paper authored by T. Yoshimoto is concerned with the relations between the limit functions in random ergodic theorems and the random parameters concomitant with the limit functions. In the paper by A. Pekin divisibility criteria are proved for class numbers of imaginary quadratic fields whose discriminant has only two prime factors. D. T. Hoa et al. study some properties of inductive limits of matrix algebras over $C^{*}$-algebras. In the paper by Y. Lan et al. some chaotic properties of discrete fuzzy dynamical systems are investigated. O. Pastravanu and M.-H. Matcovschi consider the class of matrix polytopes with a dominant vertex and the class of uncertain dynamical systems defined in discrete time, and continuous time, respectively, by such polytopes. In the paper by C.-H. Yang, an enhanced symplectic synchronization of complex chaotic systems with uncertain parameters is studied.

Several applications of uncertain dynamical systems are addressed in six papers. The paper by A. Rincon et al. presents the controller design for a second order plant with unknown varying behavior in the parameters and in the disturbance. The paper by C. Xu et al. is devoted to a stochastic chemostat model in which the dilution rate was influenced by white noise. The long time behavior of the system is studied. N. X. Quyen et al. study a chaos-based secure directsequence/spread-spectrum (DS/SS) communication system which is based on a novel combination of the conventional DS/SS and chaos techniques. In their paper, S.-Y. Li et al. expose the chaotic attractors of time-reversed nonlinear system, further implement its behavior on electronic circuit, and 
apply the pragmatical asymptotic stability theory to strictly prove that the adaptive synchronization of given master and slave systems with uncertain parameters can be achieved. Z. Liu et al. discuss a stochastic predator-prey system with Holling II functional response. The paper by W. Weera and P. Niamsup deals with the problem of robust stability for a class of uncertain Lur'e dynamical systems, neutral systems, and systems with some sector conditions.

Gani Stamov

Haydar Akca

Ivanka Stamova 


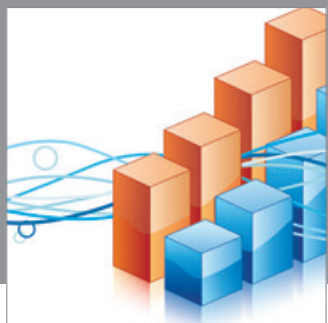

Advances in

Operations Research

mansans

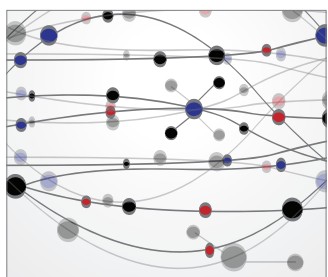

The Scientific World Journal
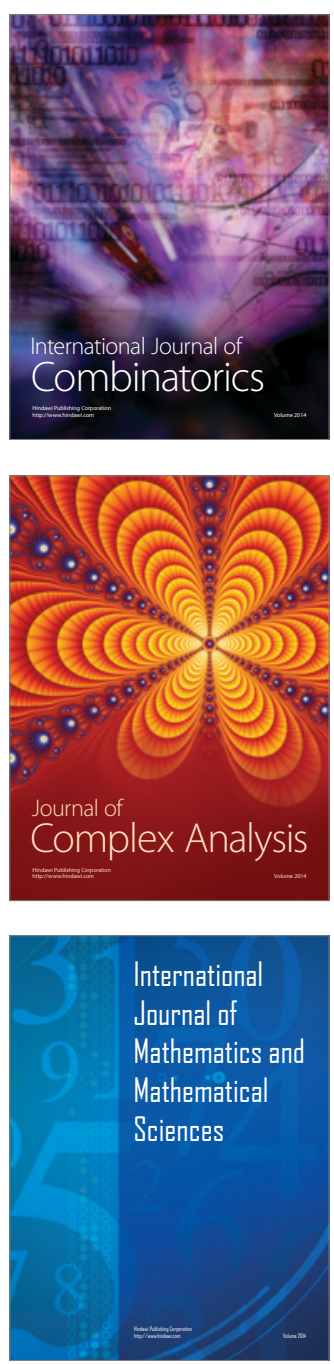
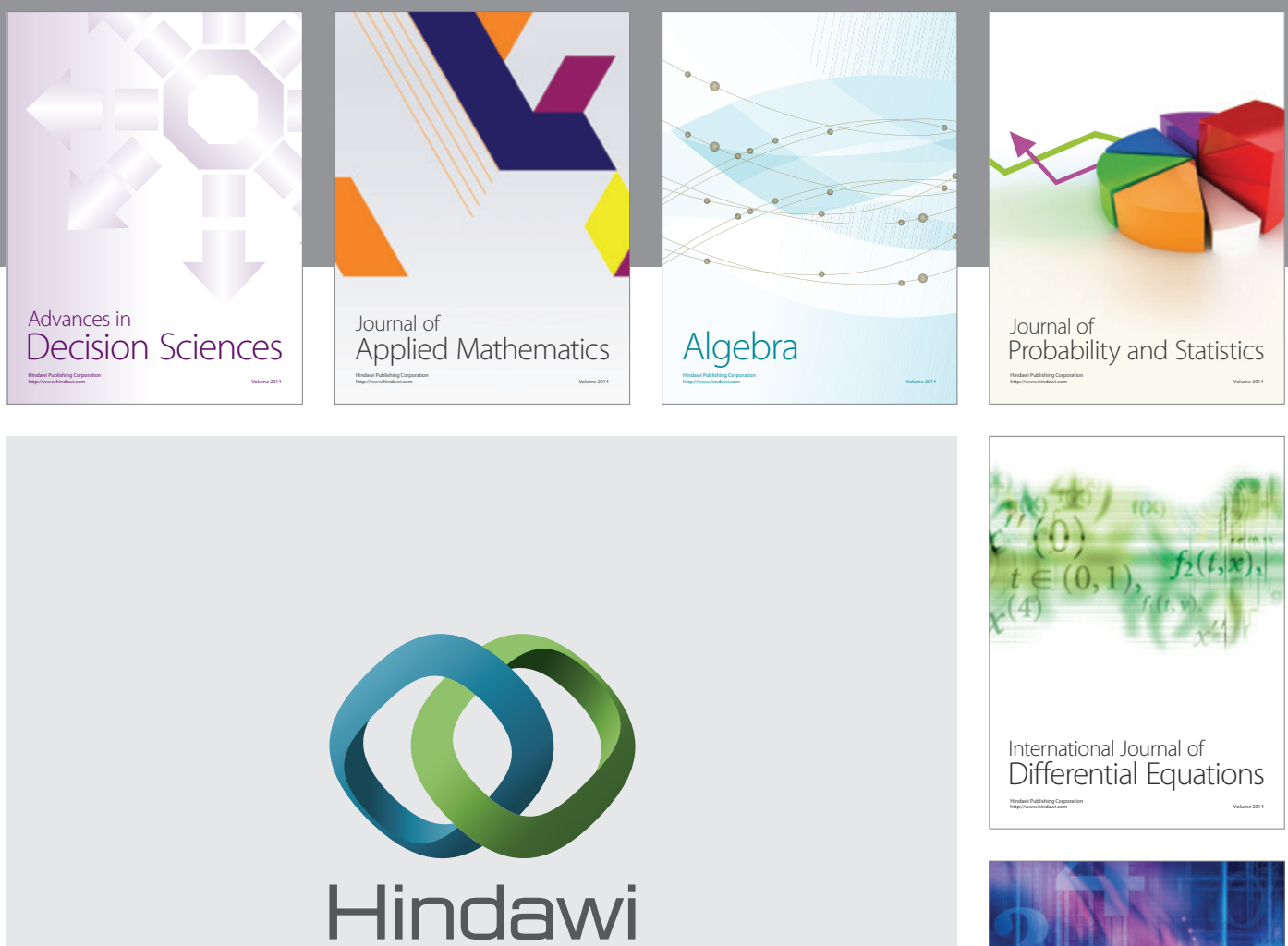

Submit your manuscripts at http://www.hindawi.com
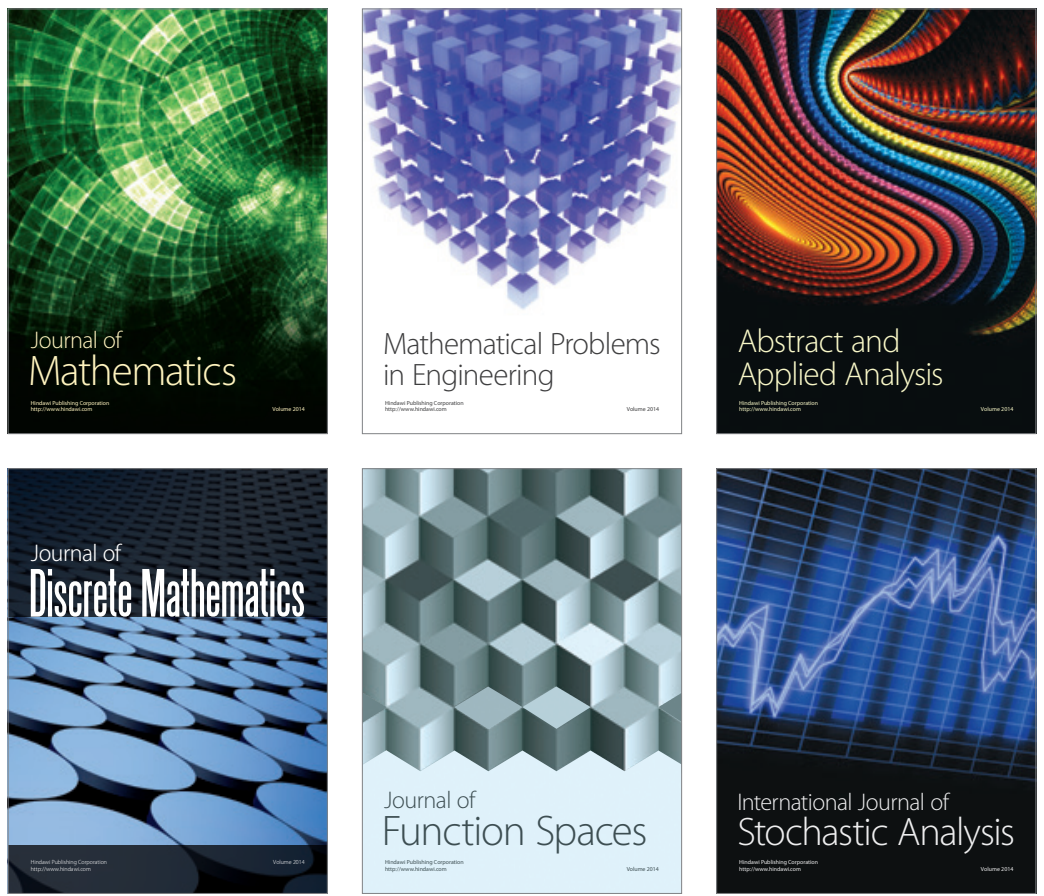

Journal of

Function Spaces

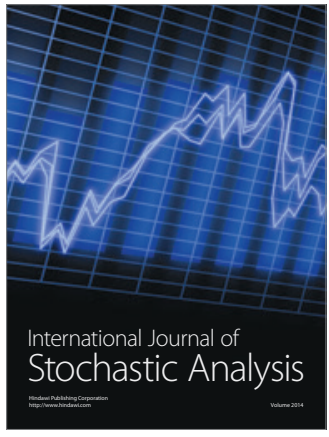

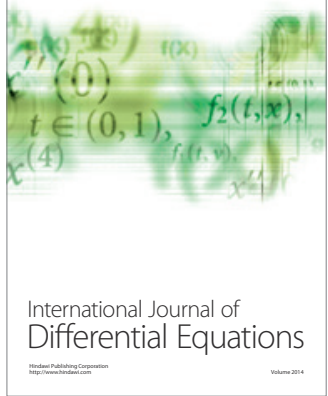
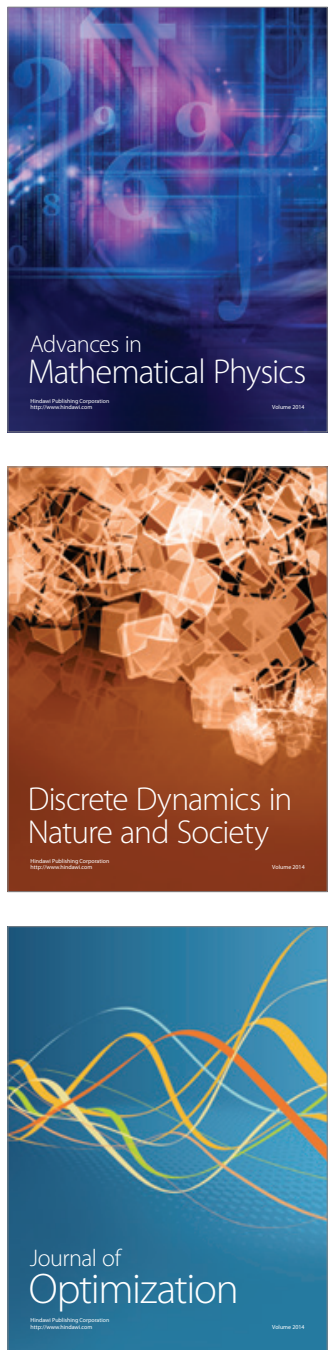